Open ACCESS

Repository of the Max Delbrück Center for Molecular Medicine (MDC) in the Helmholtz Association

http://edoc.mdc-berlin.de/15801

\title{
Autoionization in time-dependent density-functional theory
}

Kapoor, V.

This is the final version of the manuscript. The original article has been published in final edited form in:

Physical Review A

2016 JUN 08 ; 93(6): 063408

doi: 10.1103/PhysRevA.93.063408

Publisher: American Physical Society

(C2016 American Physical Society 


\title{
Autoionization in time-dependent density functional theory
}

\author{
V. Kapoor \\ Integrative Research Institute for the Life Sciences, \\ Humboldt University of Berlin, Philippstr. 13, 10115 Berlin, Germany and \\ Max Delbrück Center for Molecular Medicine, Robert-Rössle Strasse 10, 13125 Berlin, Germany
}

(Dated: May 13, 2016)

\begin{abstract}
We compute the exact exchange-correlation potential of the time-dependent density functional theory (TDDFT) for the correlated process of autoionization. The potential develops barriers which regulates the autoionization rate. TDDFT employing known and practicable exchange-correlation potentials does not capture any autoionization dynamics. Approximate exchange-correlation potentials capturing such dynamics would necessarily require memory effects and are unlikely to be developed as will be illustrated.
\end{abstract}

PACS numbers: $31.15 . e e, 42.50 . \mathrm{Hz}, 32.80 . \mathrm{Rm}$

\section{INTRODUCTION}

Exact dynamical properties of a multi-electron system can be obtained via solving the time-dependent Schrödinger equation (TDSE). Due to the "exponential wall" [1] it is computationally very challenging to solve this equation. In fact, in the case of intense laser fields where the numerical grids need to be large, a solution of the TDSE for a three electron system seems to be the maximum to date [2]. One possible way to overcome the exponential wall is the time-dependent density functional theory (TDDFT) [3 5]. In this theory the interacting multi-particle system is mapped to a unique system of non-interacting particles having the same ground state density. The observables are constructed as functionals of the single-particle density which in turn is obtained via a solution of the non-linear single-particle equations known as the time-dependent Kohn-Sham (KS) equations. The solution of the time-dependent KS equations are the single-particle orbitals from which the single particle density can be constructed. The essential ingredient in the KS construction is the Hartree-exchangecorrelation $(\mathrm{Hxc})$ potential. In many cases an adiabatic approximation to the xc potential is sufficient. However, with currently available approximations highly correlated processes like single photon double ionization, autoionization, charge-transfer and resonant interactions (Rabi floppings, [6, [7]) are not properly incorporated.

In this work we focus on the description of autoionization in TDDFT. This process in recent years has attracted a lot of theoretical and experimental attention [8 11]. Autoionization occurs due to interaction between a discrete state and the continuum states leading to emission of one or more electrons. One of the simplest system exhibiting these states is the Helium atom. In the Helium atom certain discrete states are embedded in the continuum, these states are known as the doubly excited states. Due to the electron-electron interaction these discrete states couple to the continuum leading to emission of an electron. Previous studies of this process within TDDFT have been concentrated on the linear response regime [12]. It is well known that doubly excited states can only be described in a TDDFT treatment if the exchange-correlation kernel is frequency dependent [3]. In this work we calculate the exact exchange-correlation potential for this process beyond the linear response regime from the solution of the TDSE. Exact studies such as this have led to development of better exchange-correlation potentials in TDDFT for quite a few correlated process [13, 14]. Such studies often reveal the essential features that any exchange-correlation potential should possess to be able to describe a correlated process correctly [13 16 .

The paper is structured as follows. In Sec. (III) we review the construction of exact KS potential from the solution of the TDSE. In Sec. (III) we investigate the decay dynamics of autoionizing states by solving the TDSE. In Sec. (IV) we map the solution of the TDSE to construct the exact KS Hamiltonian and the orbital. Furthermore we construct a superposition of the two states and compute the decay dynamics within TDDFT. The features observed in the exchange-correlation potential will shed light on how such a correlated process may be described within TDDFT. We conclude in Sec. (VI). The work presented here is a part of doctoral dissertation submitted to the university of Rostock.

Atomic units $\hbar=m_{e}=|e|=4 \pi \varepsilon_{0}=1$ are used throughout unless stated otherwise.

\section{BASIC THEORY}

Consider a system of $N$ interacting electrons governed by the Hamiltonian

$$
\hat{H}(t)=\hat{T}+\hat{V}_{\mathrm{ee}}+\hat{V}(t)
$$

with, in position-space representation, the kinetic energy operator

$$
\hat{T}=\sum_{i=1}^{N}-\frac{1}{2} \frac{\partial^{2}}{\partial x_{i}^{2}}
$$


the interaction potential

$$
\hat{V}_{\mathrm{ee}}=\frac{1}{2} \sum_{i \neq j}^{N} v_{\mathrm{ee}}\left(\left|x_{i}-x_{j}\right|\right)
$$

and the external potential

$$
\hat{V}(t)=\sum_{i=1}^{N} v\left(x_{i}, t\right)
$$

We assume the interaction to be Coulombic. In onedimensional models the Coulomb-interaction is usually smoothed by a softening parameter $\epsilon>0$,

$$
v_{\mathrm{ee}}\left(\left|x_{i}-x_{j}\right|\right)=\frac{1}{\sqrt{\left(x_{i}-x_{j}\right)^{2}+\epsilon}} .
$$

We further specialize on external potentials consisting of the interaction with a (static) nucleus of charge $Z$ and a laser field $E(t)$ in dipole approximation, i.e.,

$$
v\left(x_{i}, t\right)=-\frac{Z}{\sqrt{x_{i}^{2}+\epsilon}}+x_{i} E(t) .
$$

The field-free part of the Hamiltonian can be defined as

$$
\hat{H}_{0}=\hat{H}(t=0)
$$

The eigenstates and eigenenergies of the laser fieldfree system are obtained via the solution of the timeindependent Schrödinger equation

$$
\hat{H}_{0} \Psi\left(x_{1} \sigma_{1} \cdots x_{N} \sigma_{N}\right)=\mathcal{E} \Psi\left(x_{1} \sigma_{1} \cdots x_{N} \sigma_{N}\right) .
$$

Here $\Psi\left(x_{1} \sigma_{1} \cdots x_{N} \sigma_{N}\right)$ is an antisymmetric $N$-particle eigenfunction of the space and spin variables $x_{i}, \sigma_{i}$, and $\mathcal{E}$ is its eigenenergy. In order to obtain $\Psi\left(x_{1} \sigma_{1} \cdots x_{N} \sigma_{N}, t\right)$ for $t>0$ one may solve the time-dependent Schrödinger equation (TDSE)

$$
\mathrm{i} \partial_{t} \Psi\left(x_{1} \sigma_{1} \cdots x_{N} \sigma_{N}, t\right)=\hat{H}(t) \Psi\left(x_{1} \sigma_{1} \cdots x_{N} \sigma_{N}, t\right)
$$

for a fixed initial state $\Psi_{0}\left(x_{1} \sigma_{1} \cdots x_{N} \sigma_{N}\right)$. The norm $\mathcal{N}(t)$ of the wave function is defined as $\mathcal{N}(t)=$ $\langle\Psi(t) \mid \Psi(t)\rangle$.

Now we turn our attention to the non-interacting KS system that, by construction, yields the same singleparticle density $n(x, t)$ as the interacting system. For simplicity, we assume that we are dealing with spinneutral systems. The KS Hamiltonian then reads

$$
\hat{H}_{\mathrm{KS}}([n] ; t)=-\frac{1}{2} \frac{\partial^{2}}{\partial x^{2}}+v(x, t)+v_{\mathrm{Hxc}}([n] ; x, t),
$$

where $v(x, t)$ is the external potential (6) and $v_{\mathrm{Hxc}}([n] ; x, t)$ is the Hxc potential which is a functional of the single-particle density $n(x, t)$ (for notational simplicity we do not indicate the dependence on the initial states). The two potential terms combined are called the KS potential, i.e.,

$$
v_{\mathrm{KS}}([n] ; x, t)=v(x, t)+v_{\mathrm{Hxc}}([n] ; x, t) .
$$

In what follows we assume that the external laser field is monochromatic with a period $\omega_{1}$. The time-dependent KS equation reads

$$
\mathrm{i} \partial_{t} \Phi_{p}(x, t)=\hat{H}_{\mathrm{KS}}([n] ; t) \Phi_{p}(x, t),
$$

where $\Phi_{p}(x, t)$ is the $p$-th KS orbital for the KS particle with initial state $\Phi_{p}(x, 0)$. The time-dependent oneparticle density $n(x, t)$ then is

$$
n(x, t)=\sum_{p=1}^{N}\left|\Phi_{p}(x, t)\right|^{2}
$$

In order to construct exact-xc potential from the solution of the TDSE we employ a widely used numerically exactly solvable one-dimensional model Helium atom [6, 13, 17]. In this model both electrons move along the laser polarization direction only, and the Coulomb interaction is replaced by a soft-core potential as introduced before.

The TDSE Hamiltonian of the model system thus corresponds to the Hamiltonian (1) with $N=2$ and $Z=2$. The smoothing parameter was $\epsilon=1$, as, e.g., in [6]

The initial TDSE state is chosen to be the spin-singlet ground state of the interacting system

$$
\Psi_{0}\left(x_{1} \sigma_{1}, x_{2} \sigma_{2}\right)=\Psi_{0}\left(x_{1}, x_{2}\right) \frac{1}{\sqrt{2}}\left(\left|\uparrow_{1}\right\rangle\left|\downarrow_{2}\right\rangle-\left|\downarrow_{1}\right\rangle\left|\uparrow_{2}\right\rangle\right) .
$$

Since the Hamiltonian is spin-independent, the system remains also during the dipole interaction with a laser field in a spin-singlet configuration, and we can concentrate on the symmetric spatial part $\Psi_{0}\left(x_{1}, x_{2}\right)$ of the wave function only. The TDSE (9) is solved numerically on a two-dimensional numerical grid as shown in Fig. 1 with electron coordinates $x_{1}$ and $x_{2}$ using the CrankNicolson propagator to obtain the time-dependent spatial wavefunction $\Psi\left(x_{1}, x_{2}, t\right)$. The grid is divided into various regions which demarcate the bound state atom from the singly and the doubly ionized atom. In the white box region, both the electrons are close to the nucleus at the origin thus describing the neutral Helium atom. In the yellow regions one of the electron is far from the nucleus while the second one is still close to it. Hence probability density in this region describes $\mathrm{He}^{+}$. In the blue regions both the electrons are far from the nucleus. Probability density in this region describes $\mathrm{He}^{++}$.

The ground state energy of this model Helium atom is $\mathcal{E}_{g}=-2.238$, and the first excited spin-singlet is at $\mathcal{E}_{e}=-1.705$. The linear response spectra shown in Fig. 2 is calculated according to [18]. The dominant peak is associated with the transition between the ground state and the first excited spin-singlet at $\omega=\mathcal{E}_{g}-\mathcal{E}_{e}$. Transitions to the doubly-excited states can also be seen above the 


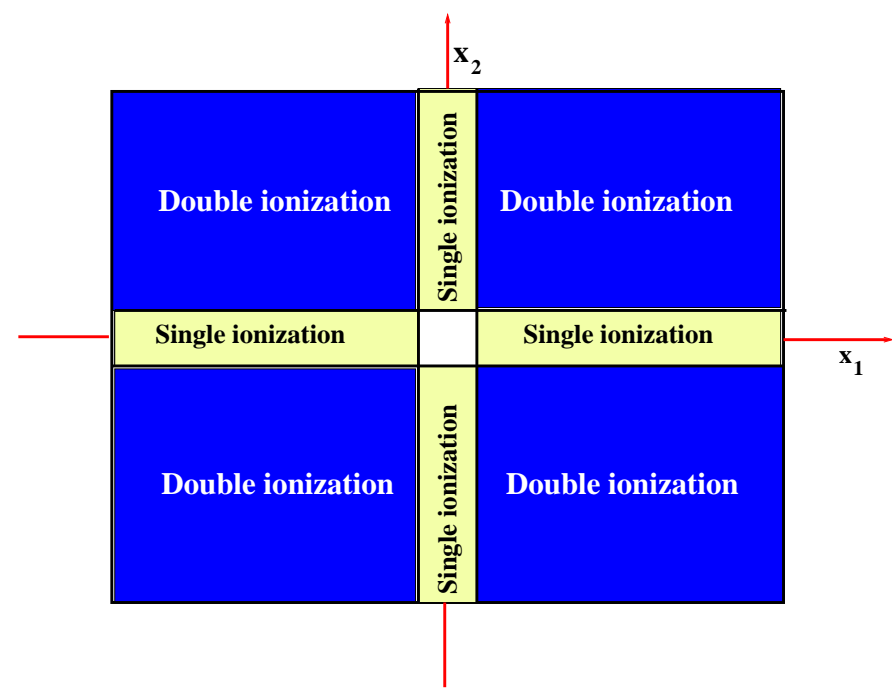

FIG. 1. (Color online) Schematic illustration of the numerical grid. Also shown are the demarcated regions which separate the bound state atom (white region) from the ionized atom (as labelled).

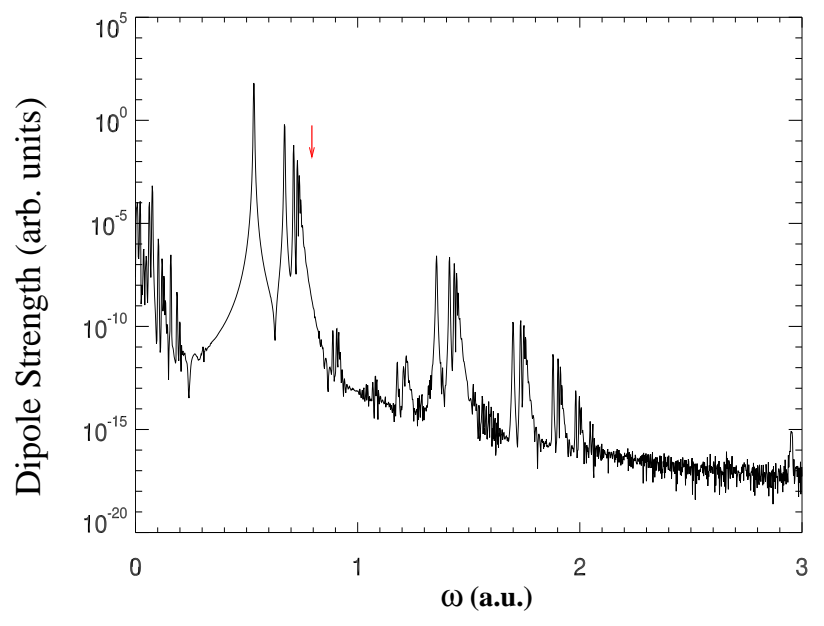

FIG. 2. (Color online) Linear response spectrum of model Helium atom obtained from the TDSE. The vertical arrow indicates the single-ionization threshold.

single-ionization threshold. Knowing the ground state energy, the energy of the doubly excited states can be determined.

Once we have obtained $\Psi\left(x_{1}, x_{2}, t\right)$ by solving the TDSE (9) we can construct the exact KS orbital and the potential following Refs. [13, 19]. In the two-electron spin-singlet case the KS wave function consists of only one spatial orbital $\Phi(x, t)$, i.e.,

$$
\begin{aligned}
& \Phi\left(x_{1} \sigma_{1}, x_{2} \sigma_{2}, t\right) \\
& \quad=\Phi\left(x_{1}, t\right) \Phi\left(x_{2}, t\right) \frac{1}{\sqrt{2}}\left(\left|\uparrow_{1}\right\rangle\left|\downarrow_{2}\right\rangle-\left|\downarrow_{1}\right\rangle\left|\uparrow_{2}\right\rangle\right) .
\end{aligned}
$$

The KS orbital can be written as

$$
\Phi(x, t)=\sqrt{n(x, t) / 2} \mathrm{e}^{\mathrm{i} S(x, t)},
$$

where $n(x, t)$ is the exact particle density and $S(x, t)$ is the exact phase of the $\mathrm{KS}$ orbital. The expression for the phase in terms of density is given by the continuity equation as [19, 20]

$$
-\partial_{x}\left[n(x, t) \partial_{x} S(x, t)\right]=\partial_{t} n(x, t) .
$$

Equation (12) can be inverted to write the KS potential in terms of the KS orbital as [19]

$$
\begin{aligned}
& v_{\mathrm{KS}}(x, t)=\frac{\mathrm{i} \partial_{t} \Phi(x, t)+\frac{1}{2} \partial_{x}^{2} \Phi(x, t)}{\Phi(x, t)} \\
& =\frac{1}{2} \frac{\partial_{x}^{2} \sqrt{n(x, t)}}{\sqrt{n(x, t)}}-\partial_{t} S(x, t)-\frac{1}{2}\left[\partial_{x} S(x, t)\right]^{2} .
\end{aligned}
$$

The imaginary part of the potential is zero due to the continuity equation (16). The density $n(x, t)$ and the phase $S(x, t)$ are computed from $\Psi\left(x_{1}, x_{2}, t\right)[13$, and by the above construction we obtain the exact KS potential. Such a straightforward construction is possible only if we have a single spatial orbital. In the general case of several KS orbitals one would need to employ a computationally more demanding fixed-point method, as demonstrated in Refs. 21, 22].

\section{A. Numerical considerations}

It is necessary to choose the grid parameters appropriately to ensure we obtain converged results. To obtain a good spatial resolution we choose the grid spacing in both dimensions to be 0.1 and the time step is chosen to be 0.025 to ensure convergence using Crank-Nicolson propagator. The time-step has to be smaller compared to the chosen spatial resolution to guarantee convergence as has been documented in Ref. [23]. As a test we perform imaginary time-propagation with the above parameters to obtain the ground state of our model Helium atom for a numerical grid of 400 grid points in both the dimensions and then do a real-time propagation with zero field intensity and compare the ground state population at the end of the real-time propagation with the initial ground state population after a simulation time of 10000 real-timesteps. We find that for the above parameters we obtain a difference between the initial ground state population and the ground state population at the end of the real-time propagation to be of the $\mathcal{O}\left(10^{-9}\right)$, which ensures the stability of the propagation. Since we study decay of autoionizing states by solving the exact TDSE, such a convergence test is essential to validate the results presented in this paper. 


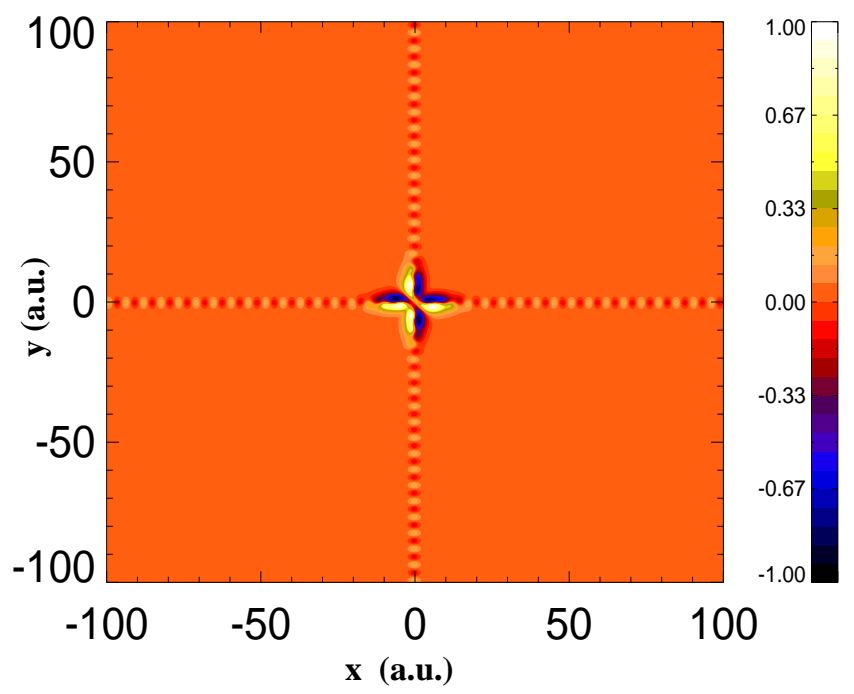

FIG. 3. (Color online) The lowest autoionizing state wave function $\mathrm{AI}_{1}$ (real-part), corresponding to $\mathcal{E}_{c}=-0.884$. The extended outgoing wave is a feature of such doubly excited states.

\section{DYNAMICAL WAVEPACKET EVOLUTION FROM THE TDSE SOLUTION}

To study the autoionization dynamics, we select three autoionizing states. The first one is the lowest lying spin-singlet state $\mathcal{E}_{\mathrm{AI}_{1}}=-0.884$ just above the single-ionization threshold. The second state is the second lowest lying spin-singlet state $\mathcal{E}_{\mathrm{AI}_{2}}=-0.816$. The third state is a higher lying spin-singlet autoionizing state with $\mathcal{E}_{\mathrm{AI}_{3}}=-0.538$ which may decay to a state with an ion in the ground state plus a "fast" electron or to a state with the ion in an excited state plus a "slower" electron. We compute these autoionizing states via the spectral method [25]. The two lowest-lying autoionizing states are shown in Fig. 3 and Fig. 4 . One of the degenerate states of the higher-lying autoionizing state is shown in Fig. 5 .

To study the decay dynamics we start with the ground state and resonantly couple it to the autoionizing state $\mathrm{AI}_{1}$. The laser pulse is a trapezoidal laser pulse with 2 cycle ramp up and down and 96 cycles of constant period. After the laser pulse is switched off the wave function at the end of the laser pulse is propagated further with only the field-free part of the Hamiltonian (see Sec. II). In Fig. 6] we plot the wavepackets in a single-ionization channel at different time instances and observe that the center of the wavepacket moves with momentum $k_{\mathrm{AI}_{1}}$ of the emitted electron. The kinetic energy of the emitted electron $\frac{k_{\mathrm{AI}_{1}}^{2}}{2}$, is dictated by the energy conservation,

$$
\mathcal{E}_{\mathrm{AI}_{1}}=\mathcal{E}_{\mathrm{He}_{\mathrm{g}}^{+}}+\frac{k_{\mathrm{AI}_{1}}^{2}}{2} .
$$

In the above equation the bound ionic state can only be

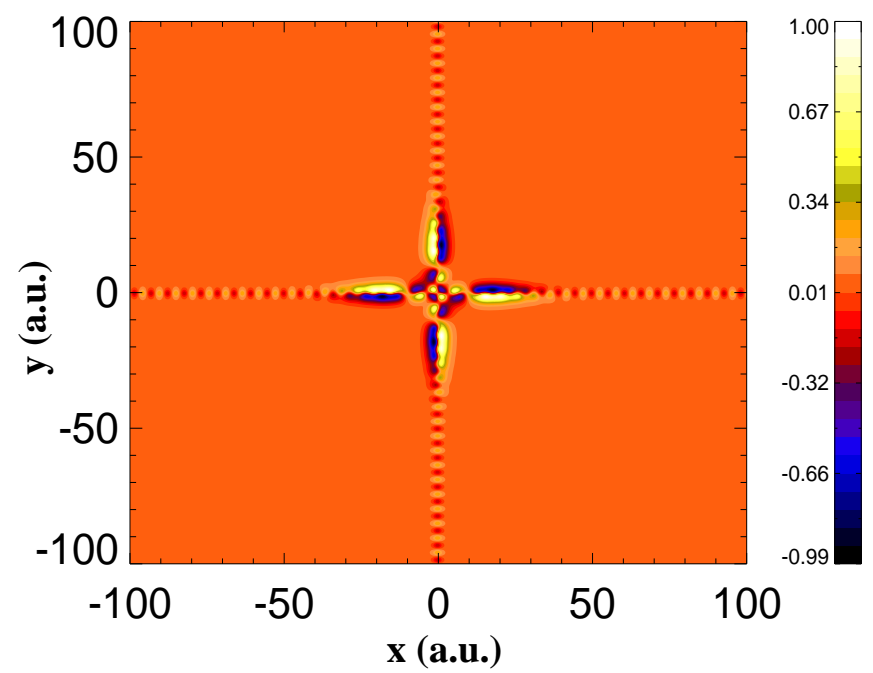

FIG. 4. (Color online) The lowest autoionizing state wave function $\mathrm{AI}_{2}$ (real-part), corresponding to $\mathcal{E}_{c}=-0.816$. The extended outgoing wave is a feature of such doubly excited states.

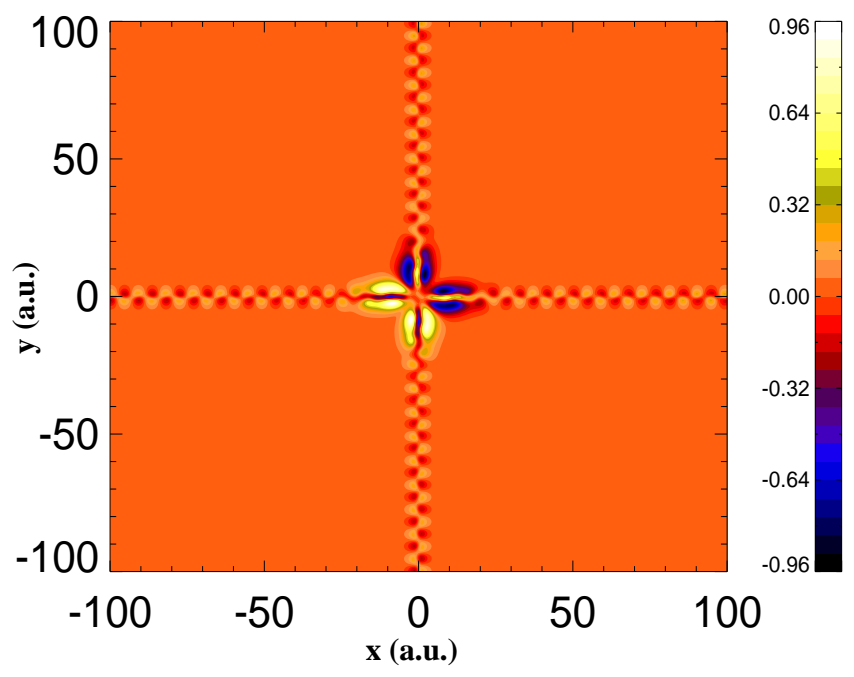

FIG. 5. (Color online) The autoionizing state wave function $\mathrm{AI}_{3}$ (real-part) above the second ionization threshold, corresponding to $\mathcal{E}_{c}=-0.538$.

the ground state as marked. The first wavepacket seen is produced via both the autoionization and the photoionization, while the trailing wavepacket is produced exclusively due to autoionization. The remaining bound electron is seen to be in the node-less ground ionic state.

To study the decay dynamics of the highest-lying autoionizing state considered $\mathrm{AI}_{3}$, we again start from the ground state and couple it to the autoionizing state $\mathrm{AI}_{3}$. The laser pulse is a trapezoidal laser pulse with 2 cycle ramp up and down and 96 cycles of constant period. After the laser pulse is switched off the wave function is propagated further with only the field-free part of the 


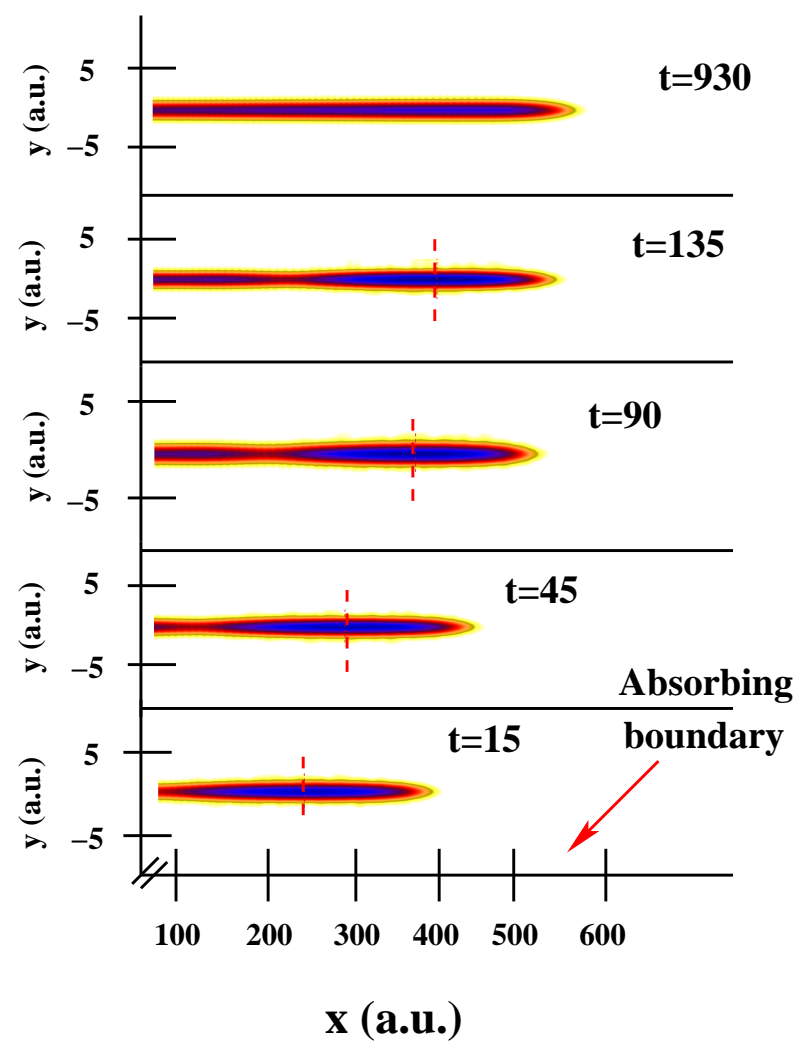

FIG. 6. (Color online) Logarithmic plot of the probability densities in the single-ionization channel at different time instants (in a.u) after the laser has been switched off. Vertical red lines indicate the classically expected position of its wavepacket with momentum $k_{\mathrm{AI}_{1}}$. Also indicated is the position of the absorbing boundary.

Hamiltonian as done previously for the autoionizing state $\mathrm{AI}_{1}$. We see that after the decay the remaining bound electron may be either in the node-less ground ionic state or the nodal first excited ionic state, as seen in Fig. 7 This follows from the following energy conservation equation,

$$
\mathcal{E}_{\mathrm{AI}_{3}}=\mathcal{E}_{\mathrm{He}_{\mathrm{g} / \mathrm{ex}}^{+}}+\frac{k_{\mathrm{AI}_{3}}^{2}}{2} .
$$

The above equation implies that the bound ionic state can now be either the ground or the excited state of the ion, which gives two possible $k_{\mathrm{AI}_{3}}$ of the emitted electron.

The center of the wavepackets move with these expected possible $k_{\mathrm{AI}_{3}}$ 's. For longer times only the probability density representing the slow photo-electron and the remaining bound electron in the excited state remains.

\section{A. Numerical considerations}

In order to determine appropriate box size for obtaining the initial autoionizing states we first accurately determine the 'target energy' of the state from the linear

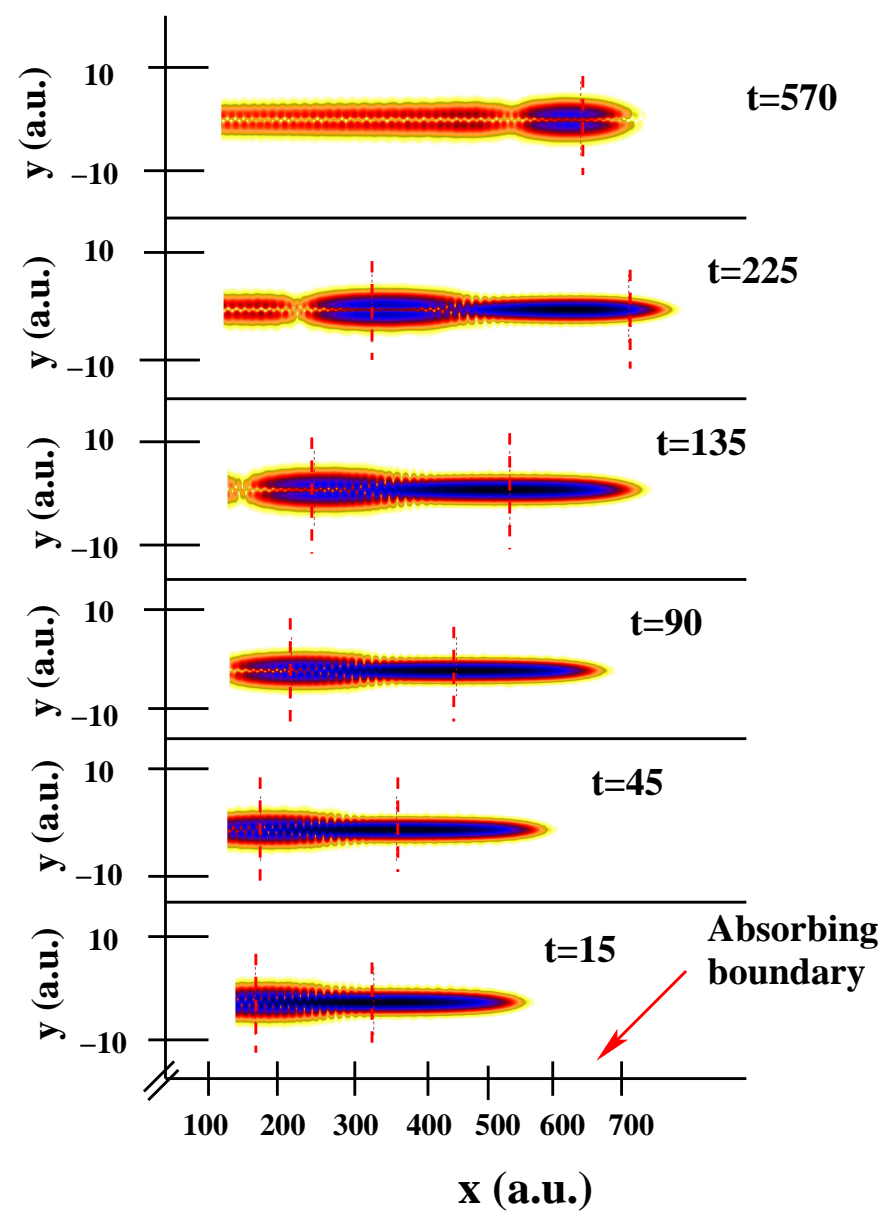

FIG. 7. (Color online) Same as in Fig 6 but for autoionizing state $\mathrm{AI}_{3}$.

response spectrum computed by kicking the ground state Fig.(2). The target energy for the first autoionizing state is the sum of $\mathcal{E}_{g}=-2.238$ and the excitation frequency of $\mathcal{E}_{\mathrm{AI}_{1}}=1.356$ (read from the linear response spectrum). Once we know the target energy of the state, the spectral method is invoked with a grid size of 2000 grid points in each direction and a grid spacing of 0.3. The spectral method converges to the 'target energy' value for up-to five decimal places for such a sufficiently large grid. If the grid size is made smaller than we chose, the value of energy becomes less accurate as we loose parts of the wavefunction near the boundary. If we make the grid bigger the energy of the wavefunction changes only after the fifth decimal place. This gives a criteria of choosing appropriate box size for the initial simulation of obtaining the autoionizing states

To study the decay of these states we re-grid the wavefunction on a grid with a grid size of $4000-5000$ grid points in each direction with the same grid spacing as before. This allows for sufficient simulation time to obtain the decay dynamics before the probability density reaches the grid boundaries. 


\section{MAPPING THE SOLUTION TO THE KS SYSTEM}

Having studied the process by solving the TDSE, we now investigate the TDDFT perspective. With just one spatial orbital it is interesting to see how such a correlated process of autoionization can be reproduced in TDDFT because the orbital, via entering the exchangecorrelation potential, has to govern its own decay. We start with the autoionizing state $\mathrm{AI}_{1}$, computed via the spectral method, and determine the exact KS potential and orbital via the previously defined technique described in section [II. In order to account for temporal and spatial loss of the density reaching the boundaries, we modify the exact KS density by including the lost parts of the probability density in the TDSE simulation because of the absorbing boundaries. Otherwise the reconstructed exact exchange-correlation potential would be incorrect. The loss terms can be computed from the grid geometry as shown earlier in Fig. 1. The total temporal loss is $(1-N(t)), N(t)$ being the TDSE norm defined in section (III). The absorbed probability density describes the $\mathrm{He}^{+}$ground state as this is the only state found in the single-ionization channels in the TDSE simulations as shown in Fig. 6. The exact $\mathrm{KS}$ density is then given by

$$
n^{\text {corr }}(x, t)=n(x, t)+(1-N(t)) n_{\mathrm{He}^{+}} .
$$

The exact KS potential is then computed with this density. The potential displays features which explain how the density decays. The essential feature is the barrier which the potential develops and through which the corresponding orbital decays via tunneling. Outside the barrier the orbital is a plane-wave with wavevector $k_{\mathrm{AI}_{1}}$, determined via the energy conservation equation (18). The height and the width of the barrier govern the decay rate whilst the binding well in the center adjusts for the correct $k_{\mathrm{AI}_{1}}$. The exact potential and the orbital for the autoionizing state $\mathrm{AI}_{1}$ at different time instants are shown in Fig. 8 Due to our density corrector step we are able to see the orbital asymptotically approaching the $\mathrm{He}^{+}$ground state shape.

The dynamics of autoionization can also be studied via resonant laser coupling of the ground and the autoionizing state. From the solution of the TDSE we compute the exact KS potential and orbital while the laser pulse is on. In Fig. 9 we see that the KS potential is simply the ground state potential of the Helium atom. With population transfer to the autoionizing state, the KS potential starts developing barriers. The height and width of the barrier are dynamically adjusted so that the KS orbital exhibits the correct decay dynamics. This allows the orbital to tunnel out with a proper decay rate.
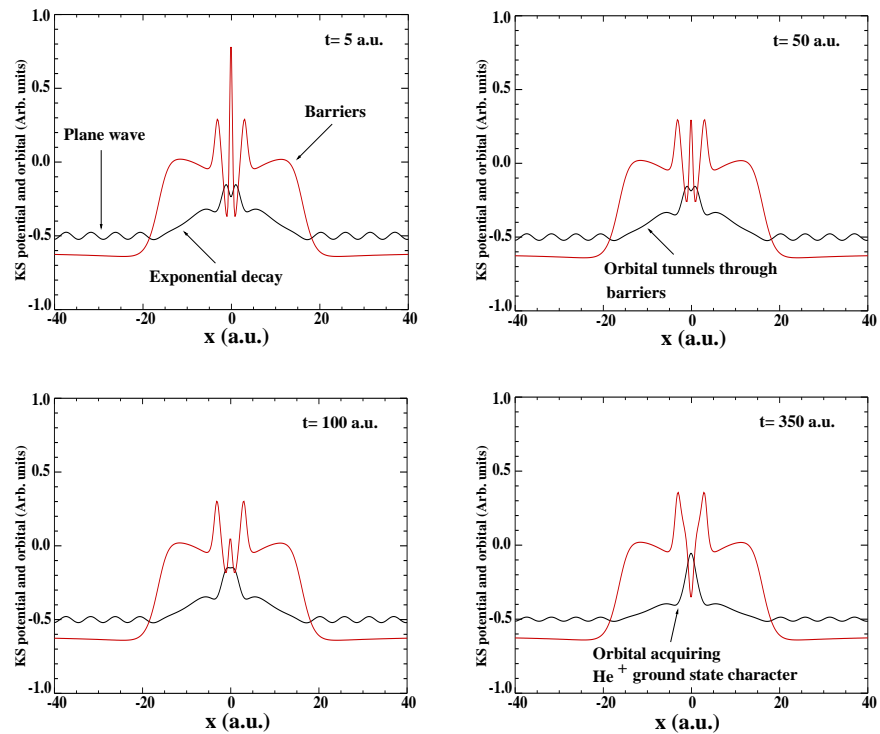

FIG. 8. The exact KS potential (red) and the corresponding orbital (black), computed from the initial autoionizing state $\mathrm{AI}_{1}$. The exact KS potential and orbital are shown at different time instances, as labelled.
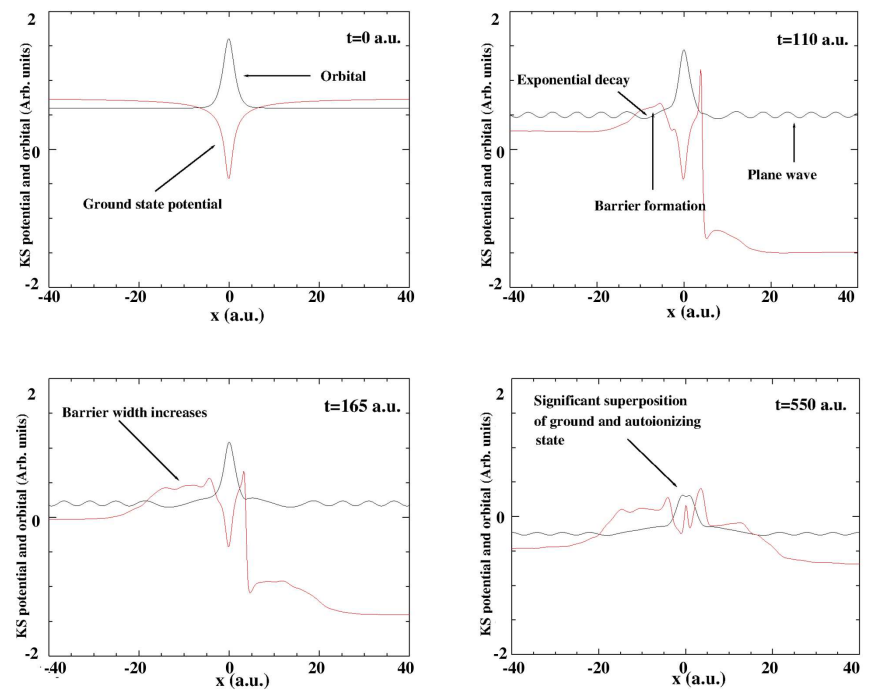

FIG. 9. The exact KS potential (red) and the corresponding orbital (black), computed for resonant laser coupling of the ground and the autoionizing state $\mathrm{AI}_{1}$. The exact $\mathrm{KS}$ potential and orbital are shown at different time instances as labelled.

\section{A. Superposition of autoionizing states}

For a superposition of two autoionizing states the $\mathrm{KS}$ potential has to control the emission of the photoelectron with two possible different wavevectors. In order to see how this is achieved by just a single spatial $\mathrm{KS}$ orbital in its corresponding exchange-correlation potential, we consider the lowest autoionizing state 


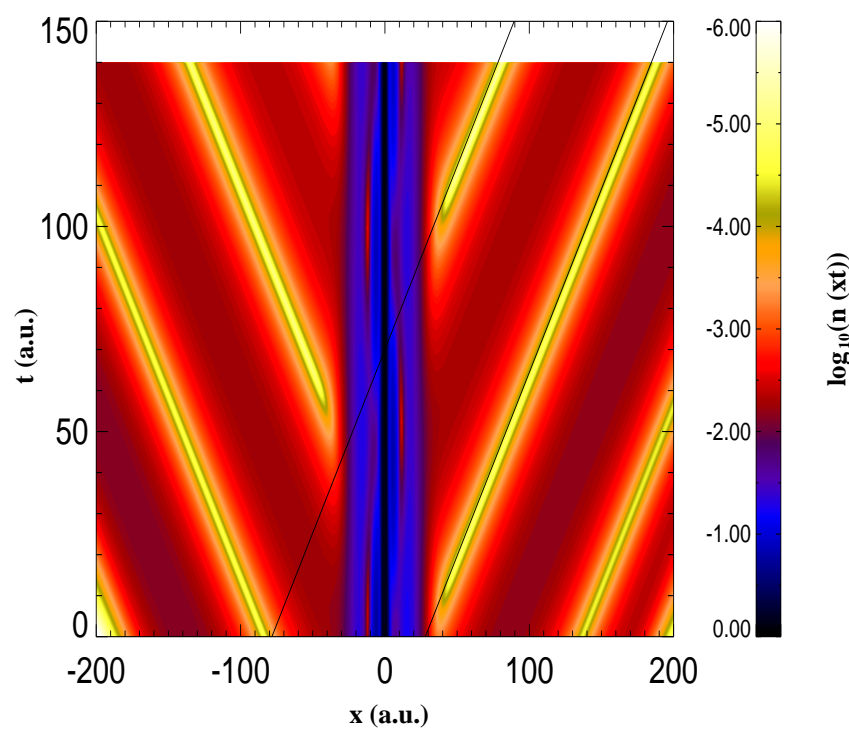

FIG. 10. (Color online) The exact KS orbital density plotted on a $\log _{10}$ scale. The oblique lines match with the over-plotted lines with the slope $\left|\frac{k_{\mathrm{AI}_{2}}-k_{\mathrm{AI}_{1}}}{\mathcal{E}_{\mathrm{AI}_{2}}-\mathcal{E}_{\mathrm{AI}_{1}}}\right|=\left|\frac{1.155-1.09}{-0.816+0.884}\right|=0.88$.

$\mathrm{AI}_{1}$ and the second lowest autoionizing state $\mathrm{AI}_{2}$. $\mathrm{An}$ equal superposition of the two states is created, and the superposed state is propagated with the field-free part of the Hamiltonian (see Sec. [II) as before [26]. The outgoing density of the orbital oscillates with the momentum $k_{\mathrm{AI}_{2}}-k_{\mathrm{AI}_{1}}$, in space and oscillates with the energy $\mathcal{E}_{\mathrm{AI}_{2}}-\mathcal{E}_{\mathrm{AI}_{1}}$, in time. The density plotted on a log scale as a function of space and time in Fig. 10 exhibits oblique lines whose slope is the ratio of momentum difference to energy difference. The exact KS potential for such a superposition of autoionizing states is shown in Fig. 11. The potential also oscillates with the respective frequencies.

The above results can be explained if we model the outgoing KS orbital as two decaying plane waves superimposed on each other. This assumption is justified as the second electron is in the same bound state for both superimposed autoionizing states. The outgoing density $n(x, t)$ in one of the single-ionization channels can then be written as

$$
\begin{aligned}
& n(x, t)=\mid \exp \left[\mathrm{i}\left(k_{\mathrm{AI}_{1}} x+\mathcal{E}_{\mathrm{AI}_{1}} t\right)-\frac{\Gamma_{\mathrm{AI}_{1}}}{2} t\right] \\
& +\left.\exp \left[\mathrm{i}\left(k_{\mathrm{AI}_{2}} x+\mathcal{E}_{\mathrm{AI}_{2}} t\right)-\frac{\Gamma_{\mathrm{AI}_{2}}}{2} t\right]\right|^{2} \\
& =\exp \left(-\Gamma_{\mathrm{AI}_{1}} t\right)+\exp \left(-\Gamma_{\mathrm{AI}_{2}} t\right)+2 \exp \left(-\frac{\Gamma_{\mathrm{AI}_{1}}+\Gamma_{\mathrm{AI}_{2}}}{2} t\right) \\
& \times \cos \left[\left(k_{\mathrm{AI}_{2}}-k_{\mathrm{AI}_{1}}\right) x+\left(\mathcal{E}_{\mathrm{AI}_{2}}-\mathcal{E}_{\mathrm{AI}_{1}}\right) t\right]
\end{aligned}
$$

Here, $\Gamma_{\mathrm{AI}_{1}}, \Gamma_{\mathrm{AI}_{2}}$ are the decay widths of the autoionizing state, $\mathrm{AI}_{1}$ and $\mathrm{AI}_{2}$, respectively. From such an expression it is clear that the density plotted on the log

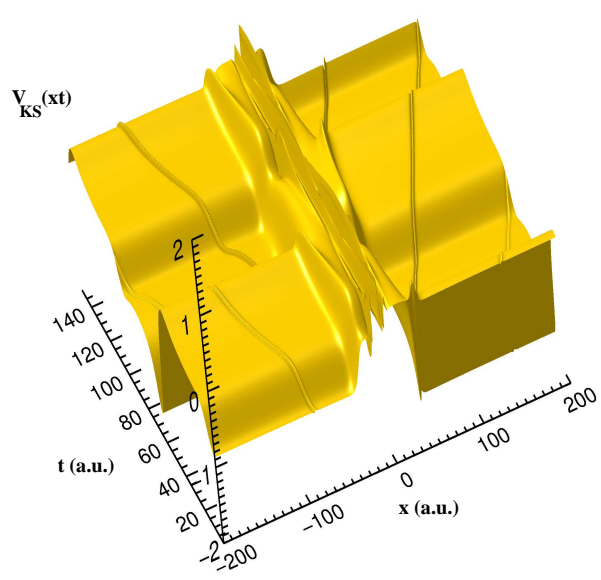

FIG. 11. (Color online) Surface plot of the exact KS potential for an equal superposition of states $\mathrm{AI}_{2}$ and $\mathrm{AI}_{1}$. The potential oscillates in time with $\mathcal{E}_{\mathrm{AI}_{2}}-\mathcal{E}_{\mathrm{AI}_{1}}$ and in space with $k_{\mathrm{AI}_{2}}-k_{\mathrm{AI}_{1}}$. Since the oscillation in space is slow, only the oblique lines of Fig. 10 are visible in the space-time plane.

scale would have oblique lines with the slope given by $\frac{k_{\mathrm{AI}_{2}}-k_{\mathrm{AI}_{1}}}{\mathcal{E}_{\mathrm{AI}_{2}}-\mathcal{E}_{\mathrm{AI}_{1}}}$.

Hence, for a superposition of two autoionizing states we obtain an oscillating barrier where the temporal oscillations result from the energy difference of the superposed state and the spatial oscillations result from the momentum difference of the emitted electron.

\section{CONSTRUCTING APPROXIMATE EXCHANGE-CORRELATION POTENTIALS}

As demonstrated above, to describe autoionization correctly requires exchange-correlation potentials which develop barriers to regulate autoionization. The barrier parameters depend on the momentum of the emitted electron which in turn depends on the energy of the autoionizing state. For modelling this process within the realm of DFT would require creating potentials which mimic such barriers, the barrier parameters such as height and width can be determined by requiring the energy of the computed state to match the experimental results. However a TDDFT study with the laser excitation is unlikely to succeed with such a modelling approach and would require development of memory-dependent exchange-correlation potentials, which would be computationally demanding and hence not practicable [27]. 


\section{CONCLUSIONS}

We did a reconstruction for the exact exchangecorrelation potentials for the highly correlated case of autoionization for TDDFT. We found that to accurately describe this process requires construction of exchangecorrelation potentials which have barriers to regulate the autoionization process. The barrier parameters such as height and width depend on the energy of the state. This implies that any approximate exchange-correlation potential would need to accurately reproduce very systemspecific features so as to have the correct barrier param- eters which ensure the correct decay rate of a particular state. This prohibits in practice the construction of universal approximations to exchange-correlation potentials for the process of autoionization. A more promising route seems to switch to a more differential basic variable instead of a single electron density, for instance time-dependent reduced density matrix theory [28].

\section{ACKNOWLEDGEMENTS}

This work was supported by the SFB 652 of the German Science Foundation (DFG). Discussions with Prof. D. Bauer are gratefully acknowledged
[1] W. Kohn, Rev. Mod. Phys. 71, 1253 (1999).

[2] C. Ruiz, L. Plaja, and L. Roso, Phys. Rev. Lett. 94, 063002 (2005).

[3] C. A. Ullrich, Time-Dependent Density-Functional Theory (Oxford University Press, 2012); M. A. L. Marques et al., Fundamentals of Time-Dependent Density Functional Theory (Springer, Heidelberg, 2012).

[4] W. Kohn and L. J. Sham. Phys. Rev. 140, A1133 (1965).

[5] E. Runge and E. K. U. Gross, Phys. Rev. Lett. 52, 997 (1984).

[6] M. Ruggenthaler and D. Bauer, Phys. Rev. Lett. 102, 233001 (2009).

[7] J. I. Fuks, N. Helbig, I. V. Tokatly, and A. Rubio, Phys. Rev. B 84, 075107 (2011).

[8] S. Gilbertson, et al., Phys. Rev. Lett. 105, 263003 (2010).

[9] Wei-Chun Chu and C. D. Lin, Phys. Rev. A 85, 013409 (2012).

[10] J. Zhao and M. Lein, New J. Phys. 14, 065003 (2012).

[11] C. Ott, et al.,Science, 340, 716 (2013).

[12] AJ. Kruegera and Neepa T. Maitra, Phys. Chem. Chem. Phys. 11, 4655-4663 (2009).

[13] M. Lein and S. Kümmel, Phys. Rev. Lett. 94, 143003 (2005).

[14] F. Wilken and D. Bauer, Phys. Rev. A 76, 023409 (2007).

[15] V. Kapoor, M. Ruggenthaler, D. Bauer, Phys. Rev. A 87, 042521 (2013).

[16] J. I. Fuks, P. Elliott, A. Rubio, and N. T. Maitra, J. Phys. Chem. Lett. 4, 735 (2013).

[17] See, e.g., R. Grobe and J. H. Eberly, Phys. Rev. Lett. 68, 2905 (1992); S. L. Haan, R. Grobe, and J. H. Eberly,
Phys. Rev. A 50, 378 (1994); D. Bauer, Phys. Rev. A 56, 3028 (1997); D. G. Lappas and R. van Leeuwen, J. Phys. B 31, L249 (1998); M. Lein, E. K. U. Gross, and V. Engel, Phys. Rev. Lett. 85, 4707 (2000).

[18] K. Yabana and G. F. Bertsch, Phys. Rev. B 54, 4484 (1996).

[19] I. DAmico and G. Vignale, Phys. Rev. B 59, 7876 (1999).

[20] M. Ruggenthaler, S. E. B. Nielsen, R. van Leeuwen, arXiv:1209.2949v2.

[21] J. D. Ramsden and R. W. Godby, Phys. Rev. Lett. 109, 036402 (2012).

[22] S. E. B. Nielsen, M. Ruggenthaler, R. van Leeuwen, Europhys. Lett 101, 33001 (2013).

[23] D. Bauer, P. Koval, Qprop: A schrödinger-solver for intense laser-atom interaction, Computer physics communications 174, (5), 396-421 (2006).

[24] Andre D. Bandrauk, Misha Ivanov, Quantum Dynamic Imaging: Theoretical and Numerical Methods, Springer, 2011.

[25] M.D. Feit, J.A. Fleck, A. Steiger, J. Comput. Phys. 47, 412 (1982).

[26] Here the density corrector step is omitted.

[27] M. Thiele, Correlated electron dynamics and memory in time-dependent density functional theory, $\mathrm{PhD}$ thesis submitted to University of Bayreuth (2009).

[28] M. Brics, D. Bauer, Phys. Rev. A 88, 052514 (2013).. 